\title{
Lessons of mortality following COVID-19 epidemic in the United States especially in the geriatrics
}

\author{
Majid Daneshfar ${ }^{\circledR}$, Nahid Dadashzadeh $^{(\oplus)}$, Mohammad Ahmadpour $^{3}{ }^{\circledR}$, Hedieh Ragati Haghi ${ }^{4}$, Vahid \\ Rahmani $^{\mathbb{E}}$, Mehdi Forouzesh ${ }^{2^{*} \mathbb{D}}$, Rohollah Valizadeh ${ }^{6}$ \\ ${ }^{1}$ Instructor, MSc in Geriatric Nursing, Faculty Member, Medical Surgical Department, School of Nursing, Gonabad University of Medical \\ Sciences, Gonabad, Iran and Student Research Committee, School of Nursing and Midwifery, Shahid Beheshti University of Medical \\ Sciences, Tehran, Iran \\ ${ }^{2}$ Legal Medicine Research Center, Legal Medicine Organization, Tehran, Iran \\ ${ }^{3}$ Department of Public Health, Maragheh University of Medical Sciences, Maragheh, Iran \\ ${ }^{4}$ Department of Radiology, Brigham and Women's Hospital, Boston, MA 02215, USA. School of Arts and Sciences, MCPHS University, \\ 179 Longwood Ave, Boston, MA 02115, USA \\ ${ }^{5}$ Department of Operating Room, Maragheh University of Medical Sciences, Maragheh, Iran \\ ${ }^{6}$ Student Research Committee, Department of Epidemiology, School of Public Health, Iran University of Medical Sciences, Tehran, Iran
}

\section{A R T I C L E I N F O}

\section{Article Type:}

Perspective

\section{Article History:}

Received: 25 June 2020

Accepted: 14 July 2020

Published online: 24 July 2020

\section{Keywords:}

COVID-19

Transmission

Mortality

Geriatric

Metropolitan

\begin{abstract}
A B S T R A C T
COVID-19 is spreading all around the world, and is considered as the most widespread infectious disease of the century. Coronavirus transmits through respiratory droplets when in close contact with the infected person. Therefore, populous places are more likely to be the source of the novel coronavirus that is threatening the health of everyone especially the geriatric population. This study aimed to compare the transmission of coronavirus between metropolitan and non- metropolitan counties as lessons of mortality (especially in geriatric) following COVID-19 epidemic. The USAFact.org public website (https://usafacts.org/visualizations) was used to determine the transmission between metro and non- metropolitan counties. In this study, four different time periods were considered for the COVID-19 incremental trend (April 1, May 1, June 1, and July 1,2020 ). The number of cases was determined per each 10,000 population. Yellow color means no case in the metropolitan county (metro). Orange color means less than 10 cases/10,000 of the population in metro. Figure and maps were used to show the objectives of the study. In metropolitan counties, the spread of COVID-19 is very fast, which is significantly different from the non-metro counties $(P<0.001)$. The results show the sharp increasing trend of infected people in metropolitan counties. In metropolitan counties the number of infected people reached 2420316 cases per 10000 populations but the number of infected people reached 231459 cases per 10000 populations. Over the four months period, the frequency of the light colors was decreased. According to the results, susceptible people especially the elderly should move to non- metropolitan counties during the COVID-19 pandemic to be less likely at risk.
\end{abstract}

Implication for health policy/practice/research/medical education:

For the elderly to remain healthy especially regarding renal parameters during COVID-19 pandemic, one way is moving to counties with low-population because populous counties are hazardous to them..

Please cite this paper as: Daneshfar M, Dadashzadeh N, Ahmadpour M, Ragati Haghi H, Rahmani V, Forouzesh M, et al. Lessons of mortality following COVID-19 epidemic in the United States especially in the geriatrics. J Nephropharmacol. 2021;10(1):e06. DOI: 10.34172/npj.2021.06.

\section{Introduction}

COVID-19 originated from China and is a potentially deadly disease that has caused widespread concern in global public health. Coronavirus is one of the most dangerous pathogens that mainly target the human respiratory tract and this virus has a high rate of transmission $(1,2)$. For instance, Wang et al showed that from January 10, to
January 24, 2020 the number of people infected with the novel coronavirus in China increased by a factor of 31.4 (3). Although the incubation period for COVID-19 is up to 14 days after exposure, most cases occur approximately 4 to 5 days after exposure. The high reproduction number (R0) of this virus has facilitated its spread worldwide (4). Due to the fact that COVID-19 causes coagulation 
disorders (5), respiratory involvement (6), kidney $(7,8)$, neurological (9) and liver diseases (10), it can be a deadly disease and it should be prevented at any cost. Research has shown that the mortality rate of this virus in the elderly and people with underlying diseases (heart disease, cancer, kidney disease and immunodeficiency) is higher compared to healthy individuals (11). The numerous number of elderly subjects aged more than 65 years are a challenge for public health. Patients with COVID-19 developed renal disease are old population with a higher mortality rate compared to young population. COVID-19 presents a big problem for elderly with long-lasting renal involvement (12). Given that COVID-19 patients with renal involvement have 5.3 times higher mortality rate, therefore the high prevalence of renal involvement is elder people can be prognostic factor for mortality $(13,14)$. Among crowded and populous places, infected people are more likely to be exposed to non-infected people. Thus, metropolitan counties are more likely to be affected by COVID-19.

\section{Objectives}

In this study, the spread of COVID-19 in populations are interpreted demographically.

\section{Methods and Materials}

Learning from health-related events can be achieved by relying on what happened to the other populations. The USAFact.org public website (https://usafacts.org/ visualizations) was used for this purpose. In this study, which is written in an interpretive way, graphical data were used for better visualization. Four different time periods were considered for the COVID-19 incremental trend. On April 1, May 1, June 1, and July 1, 2020 the prevalence of COVID-19 in metropolitan and non-metropolitan counties was examined and compared separately. In this study, COVID-19 changes can be depicted objectively and the differences in the spread rate of COVID-19 in communities were shown in a graphic manner. The number of cases was determined per 10000 populations. Yellow color means no case in metro. Orange color means less than 10 cases/10000 in metro. Brown color means equal to or more than 10 cases/10000 in metro. The colors used in the colorful maps for non-metropolitan included light blue for no cases, light green for 10 cases/10000, and dark green for equal or more than 10 cases/10 000 . So brown and dark green mean bad conditions. The trend of COVID-19 was also examined over the four months (April to July).

\section{Ethical issues}

The research followed the tenets of the Declaration of Helsinki. The authors obtained the permission by Prof. Keith Mueller, director of the Rural Policy Research Institute (RUPRI) center (on July 13, 2020) to present the data from the site http://www.public-health.uiowa.edu/ rupri./

\section{Statistical analysis}

Descriptive statistics (frequency, mean and standard deviation) and analytical statistics were used to analyse the data analysis. All the analysis was conducted using SPSS. Repeated measure ANOVA was used to assess the value of the mortality over time. $P<0.05$ was considered statistically significant.

\section{Results}

The results showed that in metro counties, the spread of COVID-19 was very fast, which significantly varies from non-metro counties. Table 1 presents the number of cases per 10000 populations in the United States in four different points of time including April 1, May 1, June 1, and July 1,2020 . Figure 1 shows a sharp increase in the umber of infected people in metro counties. It can be observed that in metro counties the number of infected people reached 2420316 cases per 10000 populations but the number of infected people reached 231459 cases per 10000 populations. The positive trend of COVID-19 between the two counties was drawn. Using Figures 2A-2D, the spread of COVID-19 in metro and non-metro counties changed the display of the maps to dark green and brown color indicating severe condition. Over the four months, the frequency of the light colors was decreased.

\section{Discussion}

We know that the spread of COVID-19 started from China which is a populous area. In the first step, we can guess the role of populous areas in the transmission of the COVID-19. Promoting health of people in the community is one of the most important pillars of the progress of societies. Health has a broad meaning and its definition is influenced by the level of awareness and perception of societies with various geographical and cultural conditions. Health is also a dynamic process and over time, its meaning will change (15). One of the important preventive actions for reducing the number of affected people with COVID-19 is social distancing (16). The importance of communicating is known in the social level because of providing the safety distance from the COVID-19 as a respiratory disease. Given that

Table 1. Comparison of the affected people by COVID-19 between metro and non-metro counties

\begin{tabular}{llllll}
\hline County & 1 April & 1 May & 1 June & 1 July & P value \\
\hline Metro cases & 198044 & 1027556 & 1652682 & 2420316 \\
Non-metro cases & 7485 & 62364 & 136230 & 231459 \\
\hline
\end{tabular}




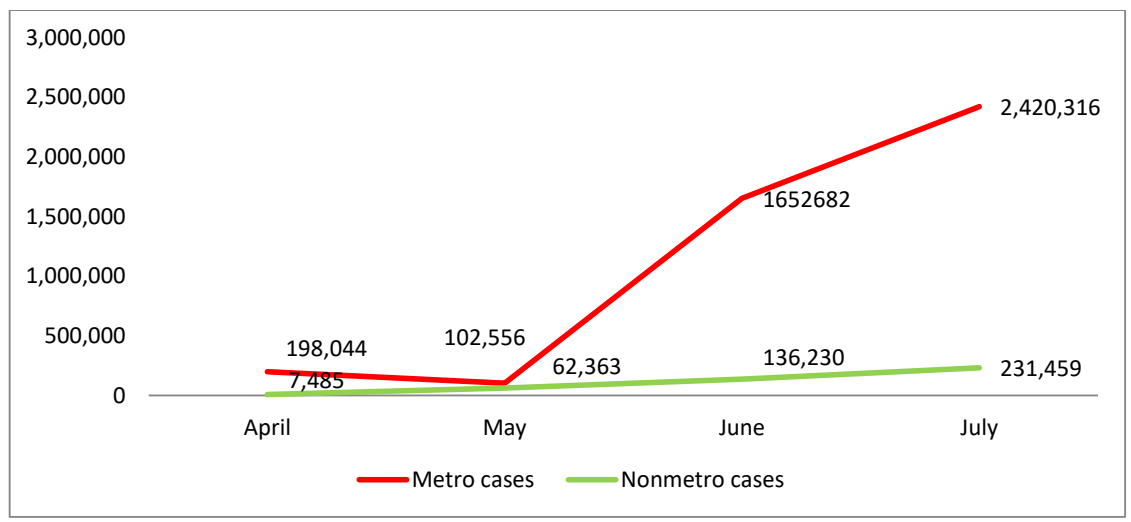

Figure 1. Trend of the infected people by COVID-19 between metro and non-metro counties.

most changes in social distancing are not mandatory, it is necessary to enhance the beliefs about disease risk and its consequences because COVID-19 is a unknown and complex disease $(17,18)$. Social distancing reduces the duration and time of exposure to this contagious virus; especially in metropolitan counties it must be observed (17). New results about transmission routes and the clinical manifestation of COVID-19 appear daily in the medical literature; it seems that the easiest and cheapest way to prevent the COVID-19 spread is avoiding crowded and populous places. Our study indicates the role of close contact and populous area in the transmission of coronavirus. As shown, the positive trend of COVID-19 between the two metropolitan and non- metropolitan counties was not equal. The spread of COVID-19 in metropolitan counties is faster than non-metropolitan counties. Elderly population is more prone to develop COVID-19 due to comorbidities. The author suggests that the transport of the people with severe conditions can be chosen as a preventive strategy, however the medical facility of the selected area is of great importance.

\section{Conclusion}

According to the results, susceptible people especially the elderly population can move to non-metropolitan counties during epidemic situations to be less likely at risk. Metropolitan counties should be kept more under observation because there are more people with close
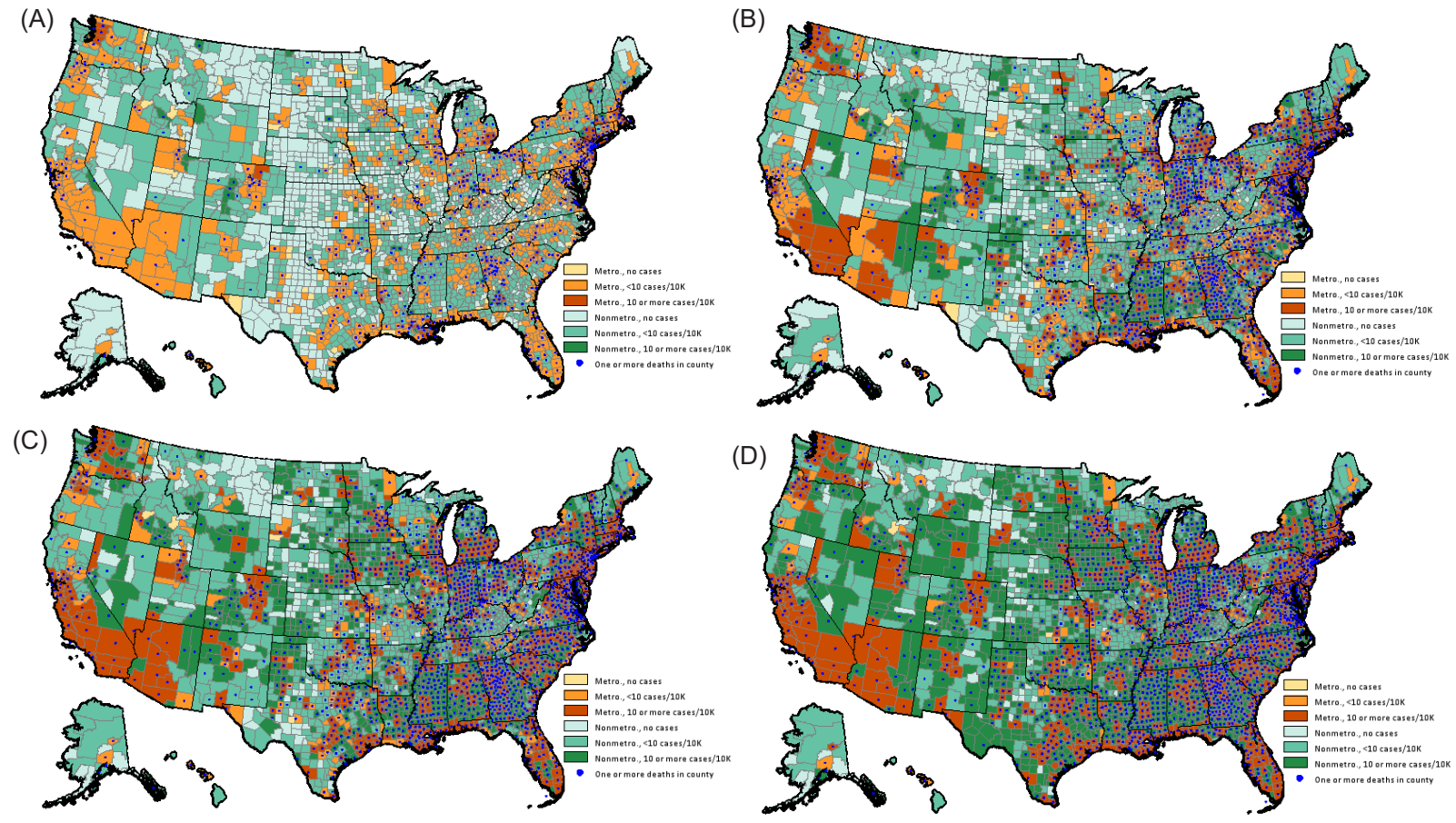

Figure 2. Map of the COVID-19 cases on (A) April 1, (B) May 1, (C) June 1, and (D) July 1, 2020. 
contact. To remain healthy during COVID-19 pandemic especially regarding renal parameters of elder patients, leaving the populous cities is a helpful strategy.

\section{Limitations of the study}

The major limitation of our study is the limited number of countries used to determine the hypothesis of the study. The results can be hypothesized but not generalized.

\section{Authors' contribution}

Study concept and design, MD; acquisition of data: MA, ND, MF; statistical analyses; MD; drafting of the manuscript: $\mathrm{HRH}, \mathrm{RV}$; analytic review of the draft: $\mathrm{HRH}$, MA.

\section{Conflicts of interest}

The authors declare they do not have any conflict of interest.

\section{Ethical considerations}

Ethical issues (including plagiarism, misconduct, data fabrication, falsification, double publication or submission, redundancy) have been completely observed by the authors.

\section{Funding/Support}

None.

\section{References}

1. Lu H, Stratton CW, Tang YW. Outbreak of pneumonia of unknown etiology in Wuhan, China: The mystery and the miracle. J Med Virol. 2020;92:401-402. doi: 10.1002/ jmv.25678.

2. Zhao S, Lin Q, Ran J, Musa G, Yang W, Wang L, et al. Preliminary estimation of the basic reproduction number of novel coronavirus (2019-nCoV) in China, from 2019 to 2020: A data-driven analysis in the early phase of the outbreak. Int J Infect Dis. 2020;92:214-7. doi: 10.1016/j. ijid.2020.01.050.

3. Wang W, Tang J, Wei F. Updated understanding of the outbreak of 2019 novel coronavirus (2019-nCoV) in Wuhan, China. J Med Virol. 2020;92:441-447. doi: 10.1002/ jmv.25689.

4. Li Q, Guan X, Wu P, Wang X, Zhou L, Tong Y, et al. Early Transmission Dynamics in Wuhan, China, of Novel Coronavirus-Infected Pneumonia. N Engl J Med. 2020;382:1199-207. doi: 10.1056/NEJMoa20013165.

5. Tang N, Bai H, Chen X, Gong J, Li D, Sun Z. Anticoagulant treatment is associated with decreased mortality in severe coronavirus disease 2019 patients with coagulopathy. J Thromb Haemost. 2020;18:1094-9. doi: 10.1111/jth.14817

6. Li X, Ma X. Acute respiratory failure in COVID-19: is it "typical" ARDS? Crit Care. 2020;24:198. doi: 10.1186/ s13054-020-02911-9

7. Ronco C, Reis T. Kidney involvement in COVID-19 and rationale for extracorporeal therapies. Nat Rev Nephrol. 2020;16:308-310. doi: 10.1038/s41581-020-0284-7.

8. Zhu J, Ji P, Pang J, Zhong Z, Li H, He C, et al. Clinical characteristics of 3062 COVID-19 patients: A metaanalysis. J Med Virol. 2020;10.1002/jmv.25884. doi:10.1002/ jmv. 25884

9. Mao L, Jin H, Wang M. Neurologic Manifestations of Hospitalized Patients With Coronavirus Disease 2019 in Wuhan, China. JAMA Neurol. 2020;77:1-9. doi: 10.1001/ jamaneurol.2020.1127.

10. Zhang C, Shi L, Wang FS. Liver injury in COVID-19: management and challenges. Lancet Gastroenterol Hepatol. 2020;5:428-30. doi: 10.1016/S2468-1253(20)30057-1

11. D’Adamo H, Yoshikawa T, Ouslander JG. Coronavirus disease 2019 in geriatrics and long-term care: the ABCDs of COVID-19. J Am Geriatr Soc. 2020;68:912-7. doi: 10.1111/ jgs. 16445

12. Coppolino G, Presta P, Nicotera R, Placida G, Vita C, Carullo N, et al. COVID-19 and renal disease in elderly patients. Geriatric Care. 2020;6(2):32-36. doi: 10.4081/ gc. 2020.9029

13. Zaim S, Chong JH, Sankaranarayanan V, Harky A. COVID-19 and Multiorgan Response. Curr Probl Cardiol. 2020;45:100618. doi: 10.1016/j.cpcardiol.2020.100618.

14. Yan Q, Zuo P, Cheng L, Li Y, Song K, Chen Y, et al. Acute kidney injury is associated with in-hospital mortality in elderly patients with COVID-19. J Gerontol. 2020. doi: 10.1093/gerona/glaa181.

15. Kwan SY, Petersen PE, Pine CM, Borutta A. Healthpromoting schools: an opportunity for oral health promotion. Bull World Health Organ. 2005; 83:677-85.

16. Andersen, Martin, Early Evidence on Social Distancing in Response to COVID-19 in the United States (April 5, 2020). Available at SSRN: https://ssrn.com/abstract=3569368. doi:10.2139/ssrn.3569368

17. Forouzesh M, Rahimi A, Valizadeh R, Dadashzadeh N, Mirzazadeh A. Clinical display, diagnostics and genetic implication of novel Coronavirus (COVID-19) epidemic. Eur Rev Med Pharmacol Sci. 2020;24:4607-15. doi: 10.26355/eurrev_202004_21047

18. Xie W, Campbell S, Zhang W. Working memory capacity predicts individual differences in social-distancing compliance during the COVID-19 pandemic in the United States. Proc Natl Acad Sci U S A. 2020;202008868. doi: $10.1073 /$ pnas.2008868117

Copyright (c) 2021 The Author(s); Published by Published by Society of Diabetic Nephropathy Prevention. This is an open-access article distributed under the terms of the Creative Commons Attribution License (http://creativecommons.org/licenses/by/4.0), which permits unrestricted use, distribution, and reproduction in any medium, provided the original work is properly cited. 